\title{
Colecistectomía laparoscópica ambulatoria: U na experiencia factible en un hospital público chileno
}

\author{
Juan Carlos Pattillo $\mathrm{S}^{\mathbf{1}}$, Rodrigo Kusanovic $\mathbf{B}^{1,2}$, Patricio Salas V1a, \\ Julio Reyes $R^{1,3}$, Ignacio G arcía-H uidobro $H^{1,3}$, Marcel Sanhueza $G^{1,3}$, \\ Alejandro Palma $D^{1}$, Sergio Báez $V^{1,2}$, Eduardo Viñuela $F^{1,3}$, \\ O rlando Friant $P^{1}$, Isabel $G$ alaz $E^{1}$, Marcos Silberman $G^{1 b}$, \\ Marco Arrese $\mathrm{J}^{3}$, Jorge Martínez $\mathrm{C}^{\mathbf{1 , 2} \text {. }}$

\section{Outpatient laparoscopic cholecystectomy. Experience in 357 patients}

Background: Laparoscopic cholecystectomy (LC) is a widely used technique in the treatment of gallstone disease. Outpatient laparoscopic cholecystectomy (OLC) is a cost/effective and safe procedure in selected patients. Aim: A pilot program of OLC was conducted in a Chilean Public Hospital to evaluate the feasibility and results, including and patients' satisfaction using OLC. Patients and Methods: Patients were eligible for OLC if they were less than 60 years old, had low anesthestetic risk (ASA I-II), normal liver function tests and an abdominal ultrasound showing gallstones or gallbladder polyps with a normal common bile duct. Results: We performed OLC in 357 patients aged $36 \pm 10$ years, $90 \%$ female. Intraoperative complications were observed in 4 (1.1\%) patients (uncontrolled bleeding in two and minor biliary tree injuries in two, both requiring conversion to the open technique). Four other patients required conversion due to anatomic reasons (overall conversion rate: $2.2 \%$ ). Ninety two percent of patients were discharged within 6 hours of the operation. Eight (2.2\%) were readmitted because of a mild acute pancreatitis $(n=1)$, biliary leakage $(n=1)$, persistent pain $(n=2)$, vomiting $(n=2)$, and retained stones $(n=2)$. Two $(0.6 \%)$ patients were re-operated. There was no mortality. Ninety-four percent of 277 patients (77.6\%), who answered a Satisfaction Survey, evaluated OLC procedure with a high degree of satisfaction. Conclusion: OLC is a safe and feasible procedure in selected gallstone patients. The procedure has good outcomes and a high degree of patient satisfaction. A wide use of OLC should reduce both direct and indirect costs of surgical treatment of gallstone disease (Rev Méd Chile 2004; 132: 429-36).

(Key Words: Cholelithiasis; Cholecystectomy, laparoscopic; Surgical procedures, laparoscopic)

Recibido el 18 de junio, 2003. Aceptado en versión corregida el 5 de enero, 2004.

${ }^{1}$ Servicio de Cirugía y Unidad de Cirugía Mayor Ambulatoria, Hospital Doctor Sótero del Río.

${ }^{2}$ Departamentos de Cirugía Digestiva y ${ }^{3}$ Gastroenterología, Facultad de Medicina, Pontificia Universidad Católica de Chile. Santiago.

anterno de Medicina. Facultad de Medicina de la Pontificia Universidad Católica de Chile.

bAfiliación actual: Unidad de Cirugía, Hospital Padre Hurtado. SSMSO.

Correspondencia a: Dr. Jorge Martínez Castillo. Departa-

mento de Cirugía Digestiva, Facultad de Medicina,

Pontificia Universidad Católica de Chile. Marcoleta 367 Ca-

silla Postal 114-D, Santiago. Fono: 56-2-354.3870.

E mail: jmartinezc@manquehue.net - jmartin@med.puc.cl 
L a colelitiasis es una patología altamente prevalente en nuestro medio. Estudios epidemiológicos recientes comunican cifras de prevalencia de hasta $20 \%$ en la población adulta masculina y entre 40 y $50 \%$ en la población femenina ${ }^{1,2}$. Además, se estima, que en nuestro país, cerca de 700 personas fallecen anualmente por complicaciones de colelitiasis $^{3}$. Por otra parte, la colelitiasis se asocia al cáncer de la vesícula biliar $^{4,5}$ y la incidencia de esta neoplasia estaría en aumento en nuestro país ${ }^{3}$. Por lo anterior, es posible afirmar que la colelitiasis constituye un problema de salud pública en Chile. A pesar de ello, su detección y tratamiento precoz en la población afectada ha sido señalada como una necesidad insatisfecha, ya que las estadísticas más recientes señalan que el aumento vegetativo de la población no se ha acompañado de un aumento proporcional en las tasas de colecistectomías ${ }^{6-8}$. Ello se relaciona con problemas operativos del sistema de salud, tales como un número limitado de camas y pabellones quirúrgicos disponibles en los hospitales del sistema público de salud. En este contexto, el diseño de estrategias que permitan ampliar la cobertura del tratamiento quirúrgico de la colelitiasis es un objetivo deseable y necesario.

Desde que Erich Mühe, en Alemania, realizara la primera colecistectomía por vía laparoscópica (CL) en $1985^{9}$, esta técnica se difundió rápidamente en Europa y Estados Unidos ${ }^{10-13}$ y es hoy considerada el método de elección para el tratamiento de la colelitiasis. En Chile, la práctica de la CL se inició en 1990, por Lizana y Sepúlveda ${ }^{14}$, para luego masificarse en el resto del país ${ }^{15,16}$. El desarmollo técnico de la cirugía mínimamente invasiva y la progresiva mayor destreza de los cirujanos que practican rutinariamente la $\mathrm{CL}$, ha estimulado el desarrollo de programas en los que la CL se efectúa en una modalidad ambulatoria, con buenos resultados y bajas tasas de complicaciones ${ }^{17}$. Nuestro grupo inició en diciembre de 1998 un programa piloto de colecistectomía laparoscópica ambulatoria (CLA), cuyos resultados preliminares en 35 pacientes comunicamos recientemente ${ }^{18}$. Los resultados iniciales indicaron una reducción, tanto del tiempo de espera por el procedimiento, como de la estadía intrahospitalaria media y un aumento de la capacidad resolutiva de nuestro hospital. El objetivo de esta comunicación es presentar la factibilidad, resultados y el grado de satisfacción del paciente de un protocolo prospectivo de CLA en un hospital público chileno en una serie de 357 pacientes consecutivos.

\section{MATERIAL Y MÉTODO}

Trabajo prospectivo, descriptivo, no controlado, a partir de un proyecto piloto en el Sistema Público de Salud iniciado en 1996 para el desarrollo de algunos tipos de cirugía mayor en la modalidad ambulatoria. Se diseñó previamente las modalidades de trabajo y organización y los protocolos específicos de trabajo clínico. Se crearon fichas de entrevistas preoperatorias, manejo intraoperatorio y controles posteriores, junto con la base de datos para la colección de información, para poder realizar un programa de colecistectomías electivas laparoscópicas y ambulatorias.

Pacientes: Se seleccionó a pacientes portadores de colelitiasis no complicada, de acuerdo a los criterios señalados en la Tabla 1. Los pacientes debían contar con apoyo familiar adecuado, estar domiciliados en el área urbana cercana al hospital, contar con teléfono y vehículo de transporte. Se excluyó a pacientes con dilatación de la vía biliar en el estudio ecográfico, aquellos con alteraciones de los exámenes bioquímicos hepáticos o que presentaran contraindicaciones para la cirugía laparoscópica. Se realizó una evaluación preoperatoria por un cirujano, un anestesista y una enfermera. A todos los pacientes se les explicó detalladamente el procedimiento y sus

\section{Tabla 1. C riterios de selección de pacientes para ser sometidos a una colecistectomía laparoscópica ambulatoria}

1. Edad $<60$ años.

2. ASA I y II compensado.

3. Ecografía ( $<3$ meses): colelitiasis y/o pólipos, sin dilatación de vía biliar.

4. Pruebas de función hepática normales.

5. Apoyo familiar compatible con el proyecto.

6. Teléfono disponible.

7. Residencia en radio urbano cercano al hospital.

8. Indice de masa corporal $<35$. 
posibles complicaciones. Posteriormente, se solicitó la firma de un consentimiento informado. La cirugía se realizó en la Unidad de Cirugía Mayor Ambulatonia (UCMA) del Hospital Base del área sur-oriente de Santiago (Hospital Dr. Sótero del Río).

Inducción anestésica y cirugía: El día de la intervención, los pacientes concurrieron acompañados por un familiar adulto a la UCMA, quien permaneció con ellos durante el postoperatorio inmediato y durante las $48 \mathrm{~h}$ siguientes en su domicilio. Todos los pacientes recibieron antibióticos profilácticos, metoclopramida $10 \mathrm{mg}$ iv, inhibidores del receptor $\mathrm{H} 2$ de la histamina (ranitidina $50 \mathrm{mg}$ iv), droperidol 0,25-0,5 mg iv. La intervención quirúrgica se realizó bajo anestesia general balanceada en base a desfluorano o intravenosa con remifentanilo y propofol. Se practicó una CL empleando una técnica estandarizada, sin colangiografía intraoperatoria. Los sitios de inserción de trócares fueron infiltrados con $40 \mathrm{ml}$ de una mezcla 50\%: 50\% con bupivacaína $0,50 \%$ y lidocaína $2 \%$ a la que se agregó $30 \mathrm{mg}$ de ketorolaco. Al finalizar la cirugía se lavó el espacio subfrénico derecho con $250 \mathrm{ml}$ de suero fisiológico y bupivacaína al 0,02\% ${ }^{15,16}$. Todas las intervenciones fueron realizadas por un equipo estable de 9 cirujanos titulares del Servicio o bajo la supervisión de algunos de ellos.

Postoperatorio: Los pacientes fueron observados durante las primeras $6 \mathrm{~h}$ del postoperatorio en la UCMA, siendo dados de alta si cumplían con los criterios de la Tabla 2, con indicación de analgesia

\section{Tabla 2. Criterios de egreso en pacientes sometidos a una colecistectomía laparoscópica ambulatoria}

1. Haber completado un período de observación mínimo de $6 \mathrm{~h}$.

2. Capacidad de deambulación autónoma.

3. Ausencia de náuseas y vómitos en las últimas $3 \mathrm{~h}$.

4. Presencia de diuresis espontánea.

5. Signos vitales estables durante todo el período de observación.

6. Dolor tratable con analgésicos orales.

7. Acompañante disponible para movilizarlo a su vehículo de transporte. por vía oral. Cada paciente recibió un instructivo detallado con indicaciones postoperatorias, una explicación de los síntomas postquirúrgicos y los signos y síntomas de alarma que debían motivar una consulta en el hospital. Además, se les entregó una hoja con el protocolo para los controles posteriores, el cual debía ser entregado en el último control, donde se les informaba del resultado de la biopsia de la vesícula extraída. Los controles postoperatorios se realizaron entre las 24 y $72 \mathrm{~h}$, a los 7-10 días y a los 30-45 días siguientes, cuando se realizaba el alta definitiva, la administración de una encuesta de satisfacción y el análisis de los resultados del estudio histológico de la vesícula biliar extraída. La encuesta de satisfacción evaluó si el paciente: a) se consideraba muy satisfecho (lo recomienda), b) medianamente satisfecho (bueno, pero no lo recomienda) o c) insatisfecho con su tratamiento.

Análisis Estadístico. Las variables no categóricas se expresan como promedio ( \pm desviación estándar) $( \pm \mathrm{DE})$ y se compararon mediante el test $\mathrm{t}$ de Student. Un $\mathrm{p}<0,05$ fue considerado significativo.

\section{RESULTADOS}

Entre diciembre de 1998 y mayo de 2002 se seleccionó a 357 pacientes con una edad media de 36 ( \pm 10$)$ años (rango: 16-66). Noventa porciento fueron mujeres. En 328 (91,9\%) pacientes, la indicación quirúrgica fue una colelitiasis sintomática no complicada y en $4(1,1 \%)$ pólipos vesiculares. En 25 pacientes (7\%), se pesquisó una colecistitis aguda no diagnosticada. Ello no indicó una conversión a laparotomía.

El tiempo operatorio fue de $58( \pm 18) \mathrm{min}$, con un rango de 30 a 120 min (Figura 1). Los pacientes que fueron dados de alta dentro del período de observación tuvieron tiempos operatorios menores: $55( \pm 14) \mathrm{min}, \mathrm{y}$ los que debieron permanecer hospitalizados, $97( \pm 19)$ min, siendo esta diferencia estadísticamente significativa ( $\mathrm{p}<0,001)$.

Se consignó $4(1,1 \%)$ complicaciones intraoperatorias: 2 eventos hemorrágicos (un desgarro del lecho hepático y un sangrado de la arteria cística lesionada accidentalmente), que requirieron con- 


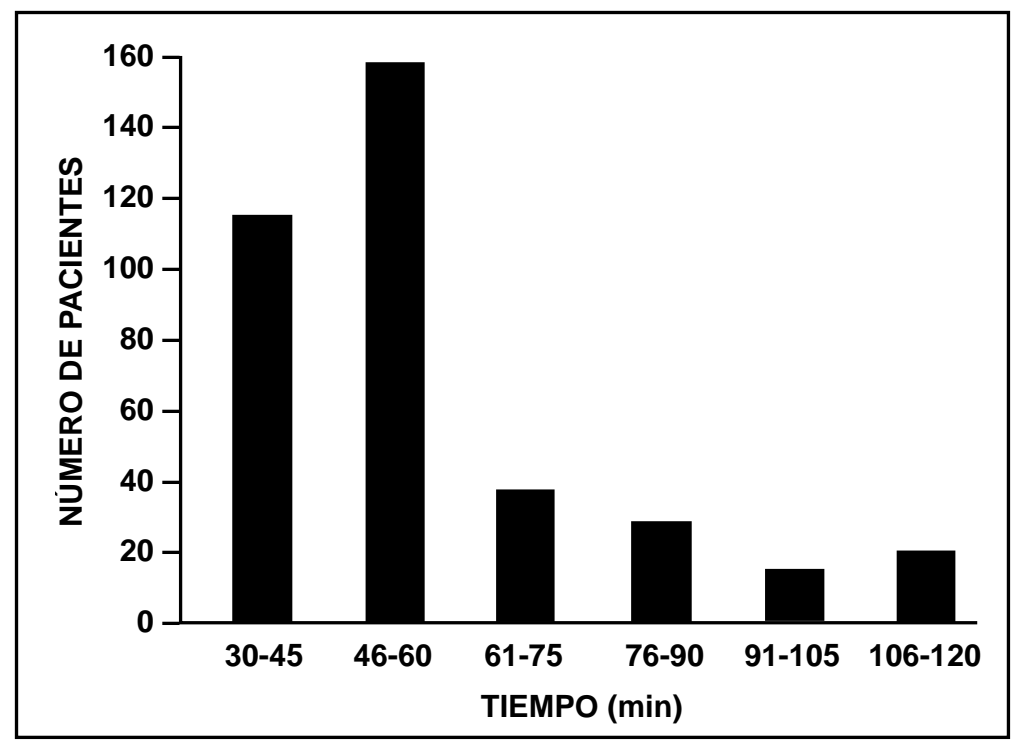

FIgURA 1. Distribución de los tiempos operatorios de 357 colecistectomías laparoscópicas ambulatorias efectuadas en la Unidad de Cirugía Mayor Ambulatoria del Hospital Dr. Sótero del Río [diciembre 1998mayo 2002].

versión a vía clásica y 2 lesiones biliares menores (un desgarro de la inserción del conducto cístico en el hepático común y una lesión parcial de un conducto sectorial posterior derecho de inserción separada en el hepático derecho), que también requirieron conversión para su identificación y reparación. Hubo otras 4 conversiones por dificultades en la identificación de la anatomía asociados a cuadros agudos (entre ellas 1 fístula colecistoduodenal y 1 síndrome de Mirizzi), completando 8 conversiones $(2,2 \%)$. Dos $(0,6 \%)$ pacientes debieron ser reoperados. Un paciente presentó un sangrado intraabdominal postoperatorio que determinó su hospitalización y una reintervención, para efectuar hemostasia. El otro paciente que requirió una reintervención, presentó un biliperitoneo aséptico, relacionado al desprendimiento de los clips del conducto cístico. En este paciente, se practicó un aseo peritoneal y se suturó el muñón cístico con buenos resultados. La morbilidad postoperatoria en 7 pacientes $(2,0 \%)$ se resume en la Tabla 3. No hubo mortalidad postoperatoria en la presente serie de pacientes.

Trescientos veintiocho pacientes $(91,9 \%)$ fueron dados de alta luego de cumplir el período de observación de $6 \mathrm{~h}$ y los criterios señalados en la Tabla 2. Otros $4(1,1 \%)$ pacientes permanecieron en observación por 2 a 3 h más, no requiriéndose su permanencia en el hospital (93\% de la serie).
Tabla 3. M orbilidad postoperatoria en 357 pacientes sometidos a una colecistectomía laparoscópica ambulatoria

\begin{tabular}{|lcc|}
\hline Morbilidad & $\mathrm{n}$ & $(\%)$ \\
\hline Infección herida operatoria (umbilical) & 1 & $(0,3)$ \\
Pancreatitis aguda leve & 1 & $(0,3)$ \\
Colédoco litiasis residual & 2 & $(0,6)$ \\
Hemoperitoneo (reoperado a las 8 h) & 1 & $(0,3)$ \\
Biliperitoneo (reoperado a los 7 días) & 1 & $(0,3)$ \\
Hernia incisional umbilical (9 meses)* & 1 & $(0,3)$ \\
Total & 7 & $(2,0)$ \\
\hline
\end{tabular}

*Este paciente fue posteriormente intervenido de su hernia incisional también en forma ambulatoria.

Veinticinco (7\%) pacientes debieron ser hospitalizados en nuestro Servicio de Cirugía. Diez $(2,8 \%)$ fueron dados de alta al día siguiente, antes de cumplir $24 \mathrm{~h}$ y 15 (4,2\%) debieron permanecer hospitalizados por más de un día. Entre ellos se cuentan los 8 pacientes convertidos a laparotomía, uno de los dos pacientes reintervenidos y los que permanecieron en observación por indicación del cirujano tratante. La Tabla 4 detalla las causas de retención de pacientes en el Hospital. 
Hubo 8 (2,2\%) readmisiones. Dos pacientes ingresaron por coledocolitiasis residual en la tercera semana y segundo mes del postoperatorio. A ambos se les practicó una colangiopancreatografía retrógrada endoscópica (CPRE), una de ellas fue normal, interpretándose el cuadro como un cólico coledociano con expulsión espontánea del cálculo y la otra confirmó una litiasis residual, realizándose una papilotomía y extracción del cálculo sin incidentes. Otro paciente reingresó por dolor persistente y aparición de vómitos frecuentes en el tercer día postoperatorio. Su estudio demostró una pancreatitis aguda edematosa, por lo que requirió una hospitalización de 10 días. Posteriormente, una CPRE no demostró coledocolitiasis residual. Otros 2 pacientes reingresaron por dolor abdominal persistente y otros 2 por emesis iterativa. Estos 4 enfermos reingresaron entre las 24 y $48 \mathrm{~h}$ del postoperatorio y su observación clínica, estudios bioquímicos y por imágenes, no demostraron complicaciones. El último reingreso de la serie correspondió al paciente con biliperitoneo ya citado.

Doscientos setenta $(77,4 \%)$ pacientes de los 349 sometidos a CLA, se presentaron al último control postoperatorio y contestaron la encuesta de satisfacción antes del alta definitiva. El análisis de las respuestas indicó un alto grado de satisfacción en $254(94,1 \%)$ pacientes. Otros 11 (3,2\%), se declararon medianamente satisfechos y hubiesen deseado permanecer por lo menos una noche en el hospital por lo que no recomiendan esta modalidad de atención.

El estudio histológico de la vesícula mostró displasia leve en $1(0,3 \%)$ paciente y en los 25 (7\%) pacientes con colecistitis aguda confirmó este diagnóstico (colecistitis crónica reagudizada) (Tabla 5).

\section{DisCUSIÓN}

En la práctica de la medicina actual la relación costo/efectividad de un procedimiento es un factor determinante en la aplicación de éste a mayor escala ${ }^{19}$. Hoy en día, la CL es el método de elección en el tratamiento de la colelitiasis, con claras ventajas frente a la cirugía abierta en términos de morbilidad y estadía hospitala-

\section{Tabla 4. C ausas de retención hospitalaria en pacientes sometidos a una colecistectomía laparoscópica ambulatoria}

\begin{tabular}{|lrc|}
\hline Causa & $\mathrm{n}$ & $(\%)$ \\
\hline Conversión a laparotomía & 8 & $(2,2)$ \\
Observación por indicación del cirujano* & 10 & $(2,8)$ \\
Observación por indicación del anestesista & 2 & $(0,6)$ \\
Dolor persistente & 2 & $(0,6)$ \\
Emesis persistente & 2 & $(0,6)$ \\
Hemoperitoneo postoperatorio & 1 & $(0,3)$ \\
Total & 25 & $(7,0)$ \\
\hline
\end{tabular}

* Generalmente debido a una cirugía laboriosa en vesículas agudamente inflamadas.

Tabla 5. Resultados del estudio histopatológico de la vesícula biliar en 357 pacientes sometidos a una colecistectomía laparoscópica ambulatoria

\begin{tabular}{|lrr|}
\hline Diagnóstico & $\mathrm{n}$ & $(\%)$ \\
\hline Colelitiasis, colecistitis crónica inespecífica & 328 & $(91,9)$ \\
Colelitiasis, colecistitis crónica reagudizada & 25 & $(7,0)$ \\
Pólipos de colesterol & 6 & $(1,7)$ \\
Adenomioma del fondo & 1 & $(0,3)$ \\
Displasia epitelial leve & 1 & $(0,3)$ \\
\hline
\end{tabular}

ria $^{14,15,20}$. Sin embargo, a pesar de que la CL representa un avance técnico significativo, la cobertura del tratamiento quirúrgico de la colelitiasis es aún insuficiente ${ }^{6,7}$. La realización de la CL en una modalidad ambulatoria en pacientes adecuadamente seleccionados, constituye una opción interesante para aumentar el número de colecistectomías en nuestro medio. Los resultados de esta serie clínica prospectiva muestran que la CLA es una técnica segura y posible de aplicar en nuestro país.

Las bases para el diseño y ejecución de un programa de CLA en nuestro centro se generaron a partir de una experiencia previa, en la cual $67 \%$ de los pacientes sometidos a una CL en un hospital público de Santiago podían, luego de una evaluación clínica por parte del médico residente de cirugía, ser enviados a su domicilio 
sin mayores complicaciones ${ }^{21}$. Esta conducta se pudo reforzar empleando una serie de medidas que disminuyen al mínimo las molestias en el período postoperatorio inmediato. Entre ellas, el empleo de infiltraciones de anestesia en las incisiones, lavado con solución con bupivacaína 22,23 y la correcta aspiración del gas del pneumoperitoneo al final de la cirugía, aseguran un apropiado control del dolor postoperatorio. Por otra parte, la prevención de las náuseas y vómitos postoperatorios son también muy relevantes para lograr el envío precoz de los pacientes a su domicilio. Ello se obtiene en forma eficaz empleando anestesia endovenosa con remifentanilo/propofol o bien anestesia balanceada con el gas desfluorano ${ }^{24}$.

La serie de CLA presentada en esta comunicación exhibe una baja incidencia de complicaciones intra y post operatorias y una reducida frecuencia de conversión a colecistectomía abierta, que la hace comparable con series similares publicadas en el extranjero ${ }^{17,25-28}$. Más de $90 \%$ de los pacientes fueron dados de alta dentro del período de observación de 6 h y más de $95 \%$ antes de las $24 \mathrm{~h}$ de la intervención. Sólo 8 de los pacientes egresados en el plazo determinado requirieron ser rehospitalizados. $\mathrm{Si}$ bien algunas de las complicaciones de la CL, tales como hemorragias o neumotórax, pueden ser detectadas en la sala de recuperación, otras, como las lesiones de la vía biliar, se pueden presentar entre 1 y 10 días después, por lo que incluso con hospitalizaciones más prolongadas pueden pasar inadvertidas si no se controlan 29,30. El control postoperatorio estricto y la motivación de los pacientes juegan un rol importante en la detección de complicaciones más tardías de la CLA, las que en nuestra serie son de ocurrencia infrecuente.

En este estudio se documentó una diferencia estadísticamente significativa en los tiempos operatorios de los pacientes que fueron dados de alta dentro del período de observación predefinido respecto de los que debieron permanecer hospitalizados. Esta diferencia se mantiene aun si se excluyen del segundo grupo los pacientes que debieron ser convertidos a colecistectomía abierta.
El tiempo operatorio ha sido señalado como un factor predictivo de la necesidad de retención hospitalaria, aunque este concepto no es universalmente aceptado ${ }^{23}$.

La selección cuidadosa de los pacientes es un aspecto importante en la planificación de un programa de CLA 30,31 . Los resultados obtenidos en esta serie reflejan, en parte, la estricta selección de pacientes jóvenes y sin otras patologías. Al no incluir a pacientes mayores, se pretende seleccionar un grupo de pacientes con patología biliar de corta data, que no han desarrollado complicaciones tardías de la colelitiasis y que no ofrecen dificultades técnicas mayores. La exclusión de pacientes con comorbilidad asociada, con riesgo de desarrollar descompensaciones de su patología de base, con estadías más prolongadas, es otro aspecto relevante en el lograr un alta precoz sin riesgo para los pacientes. Por otra parte, el grado de receptividad de los conceptos de autocuidado, por parte de los pacientes, como la existencia de una red de apoyo domiciliario adecuado, son también factores que inciden en el momento de considerar la inclusión de un paciente dado en un programa de CLA.

Finalmente, es destacable el alto grado de satisfacción con que los pacientes sometidos a CLA evaluaron el procedimiento. Ello ha determinado una mayor derivación de pacientes a nuestro centro y una operación continua del programa.

En conclusión, los resultados de este estudio permiten concluir que la CLA es una técnica que puede ser realizada en pacientes bien seleccionados de manera segura, con buenos resultados, baja morbilidad y alto grado de satisfacción de los pacientes. La aplicación a mayor escala de programas similares puede contribuir a aumentar la tasa de colecistectomías en nuestro país, por medio de una reducción del tiempo de espera para la realización de dicha cirugía. La CLA es una alternativa mínimamente invasiva ${ }^{32}$ y probablemente costo-efectiva ${ }^{25}$ para aumentar la cobertura nacional del procedimiento y permitir el tratamiento quirúrgico de la colelitiasis en la población que lo requiera. De este modo, Chile podría alcanzar estándares internacionales en esta área ${ }^{7,8}$. 


\section{REFERENCIAS}

1. Marinovic I, Guerra C, Larach G. Incidencia de litiasis biliar en material de autopsias y análisis de composición de los cálculos. Rev Méd Chile 1972; 100: 1320-7.

2. Miquel JF, Covarrubias C, Viшarroel L, Mingrone G, Greco AV, Puglew L et al. Genetic epidemiology of cholesterol cholelithiasis among Chilean Hispanics, Amerindians, and Maoris. Gastroenterology 1998; 115: 937-46.

3. Ministerio de Salud de Chile. Situación de salud Chile 2000. http://epi.minsal.cl/epi/html/frames/ frame6.htm

4. Pandey M. Risk factors for gallbladder cancer: a reappraisal. Eur J Cancer Prev 2003; 12: 15-24.

5. Serra I, Yamamoto M, Calvo A, Cavada G, Báez S, ENDOH K ET AL. Association of chili pepper consumption, low socioeconomic status and longstanding gallstones with gallbladder cancer in a Chilean population. Int J Cancer 2002; 102: 407-11.

6. Chianale J, Valdivia G, Del Pino G, Nervi F. Mortalidad por cáncer de la vesícula biliar en Chile y su relación con las tasas de colecistectomías. Análisis de la última década. Rev Méd Chile 1990; 118: 1284-8.

7. Pérez-Ayuso RM, Hernández V, González B, Carvacho C, Navarrete C, Alvarez M et al. Natural history of cholelithiasis and incidence of cholecystectomy in an urban and a Mapuche rural area. Rev Méd Chile 2002; 130: 723-30.

8. Nervi F. Cancer of the gallbladder in Chile. Rev Méd Chile 2001; 129: 979-81.

9. MüHE E. Die erste Cholecystektomie durch das Laparaskop. Langenbecks Arch Chir 1986; 369: 804-6.

10. Dubois F, Berthelot G, Levard H. Cholecystectomie par coelioscopie. Presse Med 1989; 18: 980-3.

11. Perissat J. Laparoscopic cholecystectomy, a treatment for gallstones: from idea to reality. World J Surg 1999; 23: 328-31.

12. McKeRnan JB. Origin of laparoscopic cholecystectomy in the USA: Personal experiencie. World J Surg 1999; 23: 332-3.

13. REDDICK EJ, OLSEN DO. Outpatient laparoscopic laser cholecystectomy. Am J Surg 1990; 160: 4859 .
14. Hepp J. «ntroducción» En: Hepp J, Navarrete C. Cirugía Laparoscópica. Edit: Yuri A. Sociedad de Cirujanos de Chile. Santiago, 1993: 9-10.

15. Hepp J, Ríos H, Sepúlveda R et al. Colecistectomía laparoscópica: casuística del Hospital Militar. Rev Chil Cir 1993; 45: 57-60.

16. IbáÑez L, Velasco A, López F. Colecistectomías laparoscópicas. Experiencia Clínica. Rev Chil Cir 1994; 46: 75-80.

17. LAU H, BROOKS DC. Contemporary outcomes of ambulatory laparoscopic cholecystectomy in a major teaching hospital. World J Surg 2002; 26: 1117-21.

18. Berry M, Martínez J, García-Huidobro I, Sanhueza M, Kusanovic R, Galaz E et al. Colecistectomía laparoscópica, ¿Factible en unidades de cirugía mayor ambulatoria? Rev Chil Cir 2001; 53: 299304.

19. ORLando R $3^{\text {RD }}$, Russell JC. Managing gallbladder disease in a cost-effective manner. Surg Clin North Am 1996; 76: 117-28.

20. SCOTT-CONNER CE. Laparoscopic gastrointestinal surgery. Med Clin North Am 2002; 86: 1401-22.

21. HernÁNdez F, Rodríguez O, Rodríguez E. Colecistectomía Laparoscópica Ambulatoria. Rev Chil Cir 1999; 51: 373-8.

22. Joris J, Thiry E, Paris P, Weerts J, Lamy M. Pain after Laparoscopic Cholecystectomy and Characteristics and Effect of Intraperitoneal Bupivacaine. Anesth Analg 1995; 81: 379-84.

23. Alexander DJ, Ngoi SS, Lee L, So J, Mak K, Chan $S$ ET AL. Randomized trial of periportal peritoneal bupivacaine for pain relief after laparoscopic cholecystectomy. Br J Surg 1996; 83: 1223-5.

24. Leonard IE, Cunningham AJ. Anaesthetic considerations for laparoscopic cholecystectomy. Best Pract Res Clin Anaesthesiol 2002; 16: 1-20.

25. Rosen MJ, Malm JA, Tarnoff M, Zuccala K, Ponsky JL Cost-Effectiveness of Ambulatory Laparoscopic Cholecystectomy. Surg Laparosc Endosc Percutan Tech 2001; 11: 182-4.

26. Mjaland O, Raeder J, Aasboe V, Trondsen E, Buanes T. Outpatient Laparoscopic Cholecystectomy. Br J Surg 1997; 84: 958-61.

27. Lam D, Miranda R, Hom SJ. Laparoscopic Cholecystectomy as an Outpatient Procedure. J Am Coll Surg 1997; 185: 152-5. 
28. Richardson WS, Fuhrman GS, Burch E, Bolton JS, Bowen JC. Outpatient Laparoscopic Cholecystectomy. Outcomes of 847 Planned Procedures. Surg Endosc 2001; 15: 193-5.

29. Luanos O, Jasen A, San Martin S, Sanhueza S, Tocornal J. Morbilidad y Mortalidad de la Cirugía de la Litiasis Biliar. Rev Méd Chile 1979; 107: 400-5.
30. Kumar V, Pande GK. Complications of cholecystectomy in the era of laparoscopic surgery. Trop Gastroenterol 2001; 22: 72-9.

31. BuRNEY RE, JoneS KR. Ambulatory and admitted laparoscopic cholecystectomy patients have comparable outcomes but different functional health status. Surg Endosc. 2002; 16: 921-6.

32. Fuchs KH. Minimally invasive surgery. Endoscopy 2002; 34: 154-9.

\section{Agradecimientos}

Los autores agradecen encarecidamente al personal auxiliar y de enfermería de la Unidad de Cirugía Mayor Ambulatoria del Hospital Doctor Sótero del Río, cuya dedicación y esfuerzo han permitido la realización de este proyecto. Agradecen especialmente a la señorita Nury González S., secretaria de la UCMA, por su inestimable ayuda en mantener al día la base de datos de los pacientes operados. 